\title{
Les étriers. Contribution à l'étude de leur diffusion de l'Asie vers les mondes iranien et arabe
}

Contribution to the study of their diffusion from Asia to the Iranian and Arab worlds

Los estribos. Contribución al estudio de su difusión desde Asia hacia los mundos iranio y árabe

\section{Didier Gazagnadou}

\section{(2) OpenEdition}

\section{Journals}

Édition électronique

URL : https://journals.openedition.org/tc/266

DOI : $10.4000 /$ tc. 266

ISSN : 1952-420X

Éditeur

Éditions de l'EHESS

Édition imprimée

Date de publication : 1 juillet 2001

Pagination : 155-171

ISSN : 0248-6016

\section{Référence électronique}

Didier Gazagnadou, «Les étriers. Contribution à l'étude de leur diffusion de l'Asie vers les mondes iranien et arabe », Techniques \& Culture [En ligne], 37 | 2001, mis en ligne le 20 octobre 2005, consulté le 29 septembre 2022. URL : http://journals.openedition.org/tc/266 ; DOI : https://doi.org/10.4000/tc 266

Ce document a été généré automatiquement le 29 septembre 2022.

Tous droits réservés 


\title{
Les étriers. Contribution à l'étude de leur diffusion de l'Asie vers les mondes iranien et arabe
}

\author{
Contribution to the study of their diffusion from Asia to the Iranian and Arab \\ worlds \\ Los estribos. Contribución al estudio de su difusión desde Asia hacia los mundos \\ iranio y árabe
}

Didier Gazagnadou

Je remercie l'Institut Français de Recherche en Iran (M.A.E.), qui m'a donné les moyens de mener à bien ce travail dans le cadre d'un contrat de recherche. Je remercie également $M$ Azarnouche, A Cheikh Moussa, G. Douillet, Cl. Fabre-Vassas, M. Maskhour, Z Mérat, B. Mauriac, B. Overlaet, K Zouilaï, C. Zuckerman, pour leur aide et leurs remarques.

Dans le présent article, on abordera pour l'essentiel la question de l'introduction des étriers dans les mondes iranien et arabe. La plupart des faits de diffusion étudiés par l'anthropologie soulèvent de nombreuses difficultés : la diffusion des étriers en Eurasie ne fait pas exception et s'avère être un problème très complexe; c'est pourquoi nous nous sommes surtout centrés sur son introduction au Moyen-Orient. Mais auparavant, il est nécessaire de faire le point sur les discussions qui ont suivi le travail fondamental dans ce domaine de Lynn White Jr. (1962, traduction 1969).

2 Il y a presque quarante ans, dans un stimulant article, Lynn White Jr., historien de la technologie médiévale, tentait de reconstituer l'histoire des étriers en Eurasie. Il cherchait à déterminer, d'une part, leur origine, d'autre part, leur lente et complexe diffusion vers le Moyen-Orient puis vers l'Europe. Enfin, il se proposait de mesurer l'impact socio-politique de cette invention technique sur la société occidentale. White pensait que «l'idée première » des étriers avait son origine en Inde (II ${ }^{\mathrm{e}}$ siècle av. J.-C.) mais que l'invention concrète des vrais étriers (paire en métal) était d'origine chinoise $\left(\mathrm{V}^{\mathrm{e}}\right.$ siècle ap. J.-C). Ils se seraient diffusés parmi les nomades des steppes, puis vers le Moyen-Orient et l'Europe. Les armées byzantines ne les auraient connus qu'au début du 
VIII ${ }^{e}$ siècle. Les Arabes comme les Perses ne les auraient adoptés qu'à la toute fin du VII siècle. Ce seraient les Arabes kharijites qui, pourchassés à travers la Perse par les armées califales, auraient emprunté aux nomades turcs les étriers, lesquels, selon White, venaient très récemment d'être importés du Turkestan. Enfin, ayant atteint l'Europe par diffusions successives, les étriers y auraient produit une révolution en provoquant la constitution d'un nouveau corps social : la chevalerie.

Les thèses de White ont souvent été reprises sans grands changements. Pourtant, un article de Bivar, cité par White et antérieur à son travail, proposait déjà l'hypothèse d'une invention parallèle des étriers par les nomades des steppes et les Chinois, et de leur diffusion vers Byzance, l'Europe orientale et la Scandinavie, par le biais des Avars (Bivar 1955 : 61-65). Puis en 1972, dans un nouvel article, Bivar (1972: 286-287) fait sienne la thèse de l'origine chinoise des étriers qui auraient ensuite été empruntés par les Mongols Juan-Juan puis les nomades Avars, lesquels auraient diffusé l'invention dans l'empire byzantin et l'Europe orientale. Bivar défend la thèse de l'introduction des étriers en Iran par les Arabes; ces derniers les auraient eux-mêmes empruntés aux Byzantins qui les auraient reçus des Avars (Bivar 1972: 290, n. 68). Par la suite, les thèses des quelques auteurs (Needham 1969; Haldon 1975; Littauer 1981; Herrmann 1989 ; Clutton-Brock 1992 ; Hyland 1994 ; Digard 1994 et 1987 ; Shahid 1995) qui ont plus ou moins repris la question, bien que modifiant certains points des thèses de White et de Bivar, apparaissent comme un mixte des travaux de ces deux auteurs. On y retrouve en effet l'idée d'une probable invention des étriers, soit en Chine, soit par les nomades, puis de leur diffusion vers le Moyen-Orient et l'Europe. Or, il nous semble que sur de nombreux points, et en particulier en ce qui concerne le Moyen-Orient, l'argumentation de White était très discutable et soulevait de nombreuses questions. Commençons par l'Asie.

\section{Les étriers en Inde}

White pensait, comme nous l'avons dit, que c'est en Inde qu'apparait « l'idée première de l'étrier ". Il se fondait d'une part sur une sculpture du II ${ }^{\mathrm{e}}$ siècle av. J.-C où l'on voit un cavalier dont le gros orteil est introduit dans une sangle en cuir, et d'autre part sur une pierre gravée du Ier siècle ap. J.-C, où un cavalier a les pieds tenus pat des crochets rigides qui pendent à la selle (White 1969 : 11). Deux hypothèses sont alors possibles : soit il s'agit effectivement de l'idée d'étrier mais qui ne trouve pas la forme complète de son actualisation, soit il s'agit de simples supports qui renvoient au registre cérémoniel. En effet, et contrairement à White, il ne nous paraît pas du tout évident que la sangle d'orteil ou les crochets indiens et les véritables étriers appartiennent à la même lignée technologique. Ces supports ont probablement d'abord une fonction esthétique qui consiste à donner au cavalier une élégante posture lors des processions et cérémonies royales. C'est sans doute pourquoi Deloche écrit que cela «contribue fort peu à l'équilibre » du cavalier, renvoyant ainsi ces supports à une lignée technoesthétique. La sangle d'orteil indienne, comme les crochets, peuvent, à la limite, être considérés comme le cas d'une tendance technique qui n'a pas trouvé toutes les conditions de son actualisation, d'où leur disparition sur les iconographies indiennes postérieures entre le $\mathrm{IV}^{\mathrm{e}}$ et le IX $\mathrm{IX}^{\mathrm{e}}$ siècles ap. J.-C. (Deloche $\left.1986: 23-24\right)$. C'est seulement à partir du IX ${ }^{\mathrm{e}}$ siècle que l'on trouve de vrais étriers (paire en métal) en Inde du Nord. Ils sont d'un usage répandu au $\mathrm{X}^{\mathrm{e}}$ siècle, puis partout présents au $\mathrm{XI}^{\mathrm{e}}$ siècle, et probablement 
introduits par les musulmans (Deloche 1986: 24-25). Ils s'intègrent à un dispositif guerrier, c'est-à-dire à un agencement où ils jouent un rôle important dans l'aide qu'ils apportent pour le maniement des armes lors des combats à cheval. En fonction de ces divers éléments, et même si l'on peut discuter du problème de la lignée, la sangle et les simples crochets ne semblent pas renvoyer à d'authentiques étriers: combattre à cheval avec une telle sangle d'orteil ou avec ces crochets est, du point de vue de l'efficacité, bien différent de ce que les étriers rendent possible. C'est pourquoi, nous semble-t-il, il n'y a aucun rapport, sur le plan technologique et militaire, entre les surfaix indiens et les étriers découverts en Chine et en Sibérie.

\section{Les étriers : une origine chinoise ou turco-mongole?}

5 La question de l'origine chinoise ou turco-mongole des étriers n'a pas, elle non plus, reçu de réponse définitive, la plupart des auteurs évoquant la possibilité d'une invention autant nomade que chinoise.

6 Dans un récent article sur le harnachement et l'équitation en Chine, M. Cartier (1993: 32-33), sans exclure complètement une possible origine nomade, penche plutôt pour la thèse de l'invention chinoise ${ }^{2}$, pour ce qu'il appelle des étriers au sens moderne du terme. Il y aurait en Chine des proto-étriers ou étriers de monte, constitués par un triangle suspendu à la batte d'arçon ou au quartier de la selle et seulement du côté gauche, datant " au mieux» du début du IV $\mathrm{IV}^{\mathrm{e}}$ ap. J.-C. Les premiers étriers modernes dateraient de la dynastie des Wei septentrionaux (386-532 ap. J.-C). On précisera que la première mention d'étriers de monte en Chine date de 302 ap. J.-C. et la plus ancienne paire d'étriers de 382 au Jiangsu (Des chevaux et de hommes 1995 : 15, n. 4), donc avant la dynastie des Wei septentrionaux. En tout état de cause, et quels que soient la date et le lieu de l'invention des étriers, $\mathrm{M}$. Cartier considère qu'ils ne constituent pas une révolution en équitation, du fait que depuis des siècles, les cavaleries antiques savaient parfaitement combattre sans étriers (Cartier 1993: 36). Pour nuancer sensiblement cette affirmation, nous citerons ce qu'écrit M. A. Littauer 1981 : 99) :

" The stirrup helped a more heavily armoured rider to mount a bigger horse ; it is considered to have permitted the shock encounter with couched lance and, by offering lateral support, to have encouraged the development of the sabre; it provided a top-heavy armoured warrior with more stability in the saddle, and gave archers the possibility to rise and turn in the saddle, as well furnishing them with a firmer base which to shoot. "

7 En effet, dans le combat à cheval, et selon les armes du cavalier (épée, sabre, lance, etc.), il ne fait aucun doute que disposer ou non d'étriers fait une grande différence. White (1969 : 1-2) écrivait, à juste titre :

«L'étrier, en donnant un appui latéral, en plus de l'appui qu'offraient en avant et en arrière le pommeau et le troussequin, unit étroitement cheval et cavalier et en fit une unité de combat d'une force et violence sans précédent. La main du combattant ne portait plus le coup : elle avait simplement à le diriger. L'étrier remplaça ainsi l'énergie de l'homme par la puissance de l'animal et développa les forces offensives du guerrier.»

Refuser de voir dans l'utilisation des étriers une technique qui a transformé l'histoire de la cavalerie, et donc l'histoire militaire, nous paraît discutable. Par ailleurs, pour étayer son argument en voulant l'étendre au Moyen-Orient, M. Cartier parle de la fulgurante expansion de la cavalerie légère arabo-musulmane des $\mathrm{VII}^{e}$ et $\mathrm{VIII}^{\mathrm{e}}$ siècles, 
qui eut lieu "sans étriers" (Cartier 1993: 38). Corrigeons dès maintenant cette affirmation en rappelant que l'adoption des étriers par les cavaliers arabes date du VII siècle, et d'après White lui-même (1969: 13), de la toute fin du VII ${ }^{e}$ siècle, vers 694 . Les conquêtes musulmanes se sont donc bien faites avec des montures et des selles munies d'étriers.

M. Cartier, peu favorable à l'hypothèse d'une invention nomade, laisse malgré tout la question ouverte et fournit même à trois reprises des arguments allant précisément dans ce sens. Tout d'abord, il note que la dynastie des Wei septentrionaux -sous le règne desquels apparaissent les étriers modernes- est une dynastie du nord de la Chine fondée par les Tabgatch, population d'éleveurs nomades apparentée aux Mongols. Puis il affirme que l'invention des étriers par les nomades « cadrerait mieux » avec sa fréquence dans la zone périphérique du nord. Enfin, il signale que les Mongols, qui utilisaient des étriets à larges semelles, étaient « imités en général par les militaires chinois » (Cartier 1993 : 41, n. 33). Et en effet, de nombreux éléments militent en faveur d'une invention par les nomades des steppes d'Asie centrale et/ou de Sibérie. Les plus anciens étriers en fer ont été récemment découverts dans des sépultures Hiongnu (Xiongnu) et sont datés entre le III ${ }^{\mathrm{e}}$ et le I ${ }^{\mathrm{er}}$ siècles av. J.-C. (Tseveendorj 1996: 90) ${ }^{3}$. Cette découverte n'est pas très étonnante : l'invention des étriers doit être pensée en rapport avec une civilisation du cheval (turque, mongole et autre), avec des inventions comme le pantalon, la selle (Ambtoz 1973: 94; Cartier 1993: 31) et celles des techniques de dressage du cheval -inventions des nomades des steppes d'Asie, toutes empruntées pat les armées chinoises (Gernet 1972 : 176). Ajoutons pour notre part un élément qui n'a généralement pas retenu l'attention, à savoir le rapport entre étriers et bottes. Il parait en effet difficile de séparer le problème des étriers de celui des bottes. Celles-ci, ou à tout le moins des " chaussures » renforcées, solides et pas trop souples, surtout pour les premiers types d'étriers faits d'une simple et fine tige de métal, sont une condition essentielle pour que les étriers n'occasionnent pas de blessures. Or, les nomades turco-mongols fabriquent et portent des bottes ${ }^{4}$ depuis l'Antiquité (Schiltz 1975: 37; Leroi-Gourhan 1973: 239), d'abord à cause du climat froid, puis de l'utilisation des étriers. C'est d'ailleurs peut-être l'absence de bottes qui explique la non-transformation de la sangle d'orteil indienne. En Inde, les bottes ne sont attestées, portées à cheval, qu'au XIII ${ }^{e}$ siècle (Deloche $\left.1986: 25\right)$. Il s'agit de penser le problème de l'invention et de l'emprunt des étriers en tant qu'agencement entre le technologique, le militaire et le politique, et donc de mettre en relation étriers, selles (Ambroz 1973 : 94-98; Herrmann 1989), pantalon, bottes, organisation sociale nomade, et de considérer le rapport de ce type de société à l'espace, au cheval et à la guerre. C'est pour cet ensemble de raisons qu'il faut plus probablement voir les étriers comme une invention des nomades des steppes d'Asie centrale et de Mongolie, et plus précisément de leurs métallurgistes ${ }^{5}$. Les nomades la diffusèrent lors de leurs différentes migrations (Ambroz 1973 : 91) et des guerres qu'ils livrèrent aux États sédentaires, ce qui entraîna son emprunt par la Chine, puis par la Russie des steppes et par les mondes sassanide, byzantin et arabe.

\section{Les étriers au Moyen-Orient}

10 De l'Asie orientale, les étriers allaient atteindre le Moyen-Orient. Reprenons sur ce point en détail la célèbre thèse de White. Il affirme que les armées sassan ides ne 
connaissaient pas les étriers en métal avant leur introduction par les Arabes vers la toute fin du VII siècle. Mais, comme les Arabes ne les connaissaient pas non plus, ce serait, selon lui, le général arabe Muhallab qui, en 694, pourchassant les Kharijites azraqites assez loin en Iran, aurait emprunté à ces derniers l'idée des étriers ou les étriers eux-mêmes; les Azraqites venant eux-mêmes de les emprunter en Iran où, en provenance du Turkestan, ils avaient été récemment introduits (White 1969: 12-13). Cette thèse, aussi séduisante soit-elle, ne nous paraît pas très vraisemblable, et il est étonnant que l'on y ait jusqu'à présent adhéré aussi simplement, notamment en ce qui concerne l'absence d'étriers dans la cavalerie sassanide.

11 Voyons tout d'abord la question chez les Perses. Pour démontrer que ceux-ci ne connaissaient pas les étriers, White avance plusieurs arguments. Il mentionne le célèbre plat argenté iranien du Musée de l'Ermitage, où l'on voit nettement l'étrier gauche du cavalier, mais il rejette les datations de certains auteurs en s'appuyant sur d'autres, favorables à son hypothèse ; il fait remonter ce plat au plus tôt à la première moitié du VIII ${ }^{\mathrm{e}}$ siècle, voire, selon une autre datation, au XI ${ }^{\mathrm{e}}$ siècle! (White 1969: 36, n. 93). Or, d'après de récentes publications, ce plat, qui comporte au revers une inscription en pehlevi, date plutôt du VII ${ }^{\mathrm{e}}$ siècle ou du début du VIII ${ }^{\mathrm{e}}$ siècle (Splendeur des Sassanides 1993 : 196 et 197, fig. 54). Puis, reprenant à Pelliot (1926) le fait que le persan emploie pour désigner un étrier le mot arabe rikâb (persan rékâb), White affirme qu'il y a là une preuve que la Perse n'a jamais connu les étriers avant leur adoption et leur diffusion par les Arabes. On fera simplement remarquer que le persan dont il est question - de graphie et au fort lexique arabes- est bien postérieur au VII ${ }^{\mathrm{e}}$ siècle, époque où l'arabe a déjà supplanté le pehlevi. Il convient également de signaler qu'un travail récent indique que le mot arménien désignant l'étrier, aspandak, est d'origine perse (Tanavoli 1998: 44). En effet, asb ou asp, cheval en langue iranienne, a été conservé en arménien sous la forme d'asp, et pand vient du persan band, attache ; par contraction, l'ensemble aspandak, signifie "attache de cheval», soit l'étrier en arménien (le suffixe ak ne changeant pas le sens) ${ }^{6}$.

12 Contre l'idée répandue de l'absence d'étriers dans les armées sassanides, on peut apporter d'autres indices. Premièrement, des étriers ont été découverts dans le nord de l'Iran et sont datés du VII ${ }^{\mathrm{e}}$ siècle, voire de la fin du VI ${ }^{\mathrm{e}}$ siècle, donc de l'époque sassanide (Overlaet 1993 : 93). En second lieu, l'étude de G. Herrmann (1989: 809) sur les selles sassanides et son réexamen d'une fresque de la grande grotte de Taq-i Bustan l'amènent, vu la position des jambes et des pieds de certains cavaliers, à poser la question de la présence d'étriers vers la fin de la période sassanide (p. 771). Dans cette scène de chasse, il est en effet frappant de voir les cavaliers dans une posture qui suggère fortement la présence d'étriers, contrastant avec la position des jambes pendantes et des pieds du roi qui, dans le haut de la fresque, se tient à cheval, protégé par un parasol \{cf. photo). Cette sculpture date du règne de Khosrow II (591-628) et appartient « indubitablement à l'époque sassanide tardive » (Vanden Berghe 1993 : 87). 


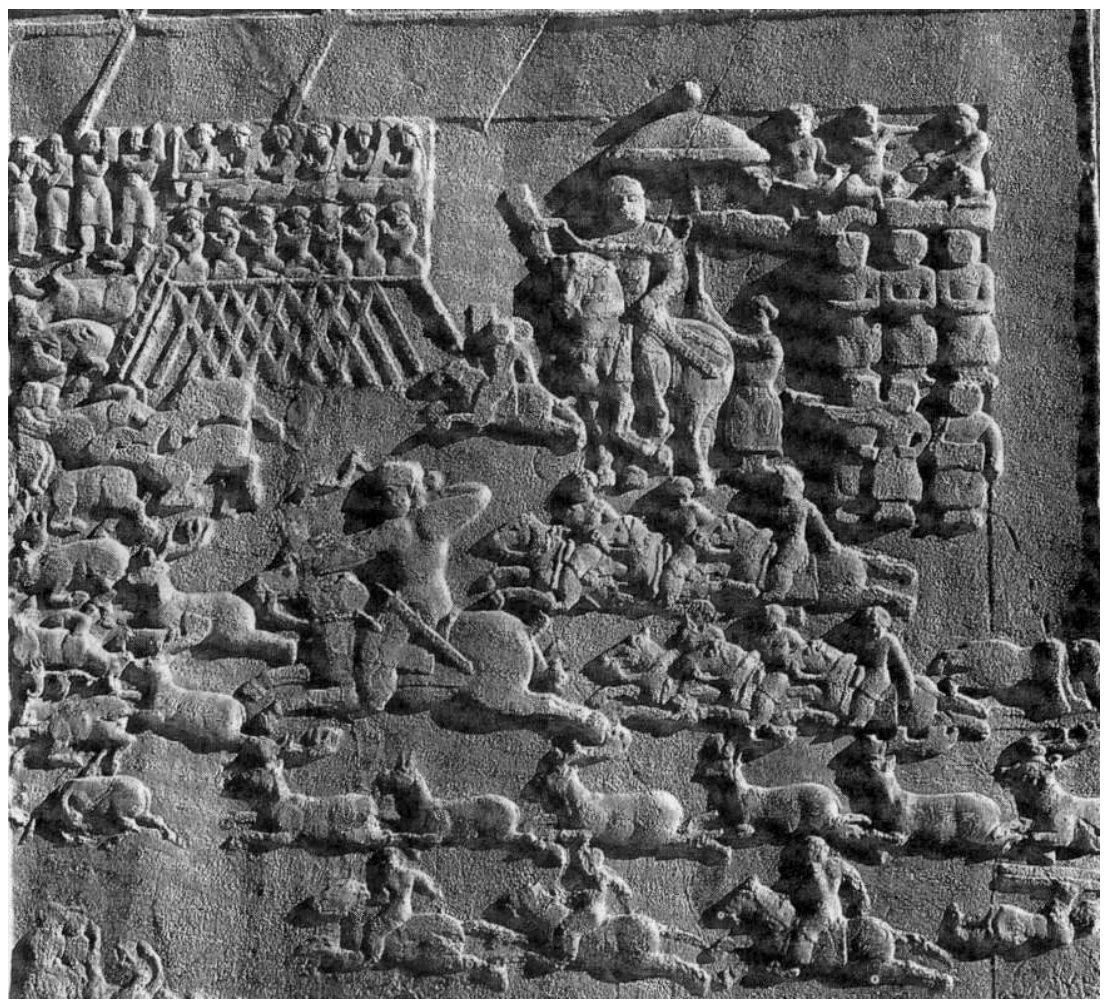

Détail de la scène de la chasse aux cerfs sur le mur droit de la Grande Grotte du Taq-i Bustan (cliché du Professeur L. Vanden Berghe)

On ajoutera par ailleurs un premier élément. Il s'agit d'un passage d'Ibn Qutayba (828-889), extrait de son Kitâb al'-arab, qui, à notre connaissance, n'a jamais été mentionné comme source. Il écrit que les Arabes montaient à cheval sans étriers et parle, par opposition, de la cavalerie sassanide, qui avait bien plus de moyens et de meilleures armes (Ibn Qutayba 1954 : 370). Il est vrai qu'il n'indique pas explicitement l'usage d'étriers dans la cavalerie perse, mais son texte le laisse entendre. En effet, dans la polémique shu'ubite, ce procédé est devenu un style littétaire où lorsqu'est enlevée une qualité à quelqu'un, elle est implicitement attribuée à la partie adverse. De même, Jâhiz (776-868) comme plus tard Ibn Qutayba parlent du goût viril des Arabes pour une monte sans étriers et les opposent à certains d'entre eux qui fréquentent de près les 'ajam (i.e. les Persans) et qui, de ce fait, sont sensibles à une autre forme de monte (Jâhiz 1968, II, t. $3: 46$; Ibn Qutayba 1968 : I. 216). Ces premiers éléments réunis nous incitent à faire l'hypothèse d'une adoption des étriers par les Sassanides au cours du premier quart du VII ${ }^{\mathrm{e}}$ siècle, voire à la fin du VI $\mathrm{e}^{\mathrm{e}}$ siècle.

14 Examinons maintenant ce qu'il en est chez les Arabes. White s'appuie sur deux sources : un extrait du Kâmil de Mubarrad (826-900) et un passage du Bayân de Jâhiz. Voyons d'abord ce que dit précisément le passage de Mubarrad :

«Les étriers des gens étaient autrefois en bois, et lorsque l'étrier du cavalier était frappé [recevait un choc], l'étrier se cassait; et si il voulait porter un coup [d'épée ou de sabre] ou un coup de lance, il n'avait plus d'appui ; c'est pourquoi Muhallab ordonna que les étriers soient fabriqués [forgés] en métal; il fut le premier à ordonner une telle fabrication » (Mubarrad s.d., deuxième partie, bâb 51 : 274-275).

Les informations livrées laissent simplement entendre que le général Muhallab serait à l'origine des étriers en métal et que les anciens Arabes ne connaissaient que ceux en bois. Mais curieusement, White, contrairement à l'affirmation de Mubarrad, conteste le 
fait que les Arabes aient pu utiliser les étriers en bois ${ }^{7}$. Quant au passage de Jâhiz, qui est une réponse aux shu'ubites, il dit ceci :

«Ils ont dit [...] lors de la guerre, vous [les Arabes] montiez les chevaux sans rien [sans selle]. Et même quand un cheval avait une selle, c'était une "selle" de dromadaire en cuir et sans étriers, alors que l'étrier est le meilleur instrument pour le guerrier, avec la lance comme avec le sabre, car il peut se mettre debout en s'appuyant dessus. » (Jâhiz 1968, vol II, t. $3: 41$ ).

Un peu plus loin, Jâhiz écrit : « Quant aux étriers, on sait qu'ils sont anciens, les étriers en métal ne furent utilisés par les Arabes qu'à l'époque des Azraqites. » (1968 II, t. 3 : 45). Ce passage indique seulement que les Arabes n'employèrent les étriers en métal qu'à l'époque des Azraqites. Or, Nâfi' b. al-Azraq, d'où vient le nom d'Azraqites, groupe kharijite radical, s'est signalé en 683 à la Mekke par sa participation à une révolte en Arabie (Wensinck 1977 ; Rubinacci 1977). Il y a tout lieu de penser que ce théoricien et son groupe existaient avant cette date. Donc, quand Jâhiz écrit "époque azraqite ", il renvoie à une période se situant entre 670-680 et 698-699, date de l'écrasement du mouvement azraqite. En fait, White fait un amalgame (sélectif) entre une information de Mubarrad affirmant que c'est à Muhallab que l'on doit les premiers étriers en métal, mais qui ne fait aucune allusion aux Azraqites, et l'information de Jâhiz qui situe leur apparition chez les Arabes à l'époque azraqite, mais qui ne mentionne pas le général Muhallab. Puis, ayant posé (un peu rapidement) l'hypothèse que ni les Perses, ni les Arabes ne connaissaient les étriers, et que les Azraqites se sont, à un moment, regroupés en Iran, White construit son interprétation d'un emprunt des étriers récemment introduits en Perse- par les Azraqites et de leur imitation par Muhallab vers 694, date de ses grandes opérations anti-azraqites en Iran. Remarquons par ailleurs que, sur cette récente introduction en Iran, White ne nous livre aucun argument qui justifie une telle interprétation. D'autre part, disons tout de suite que nous ne retenons aucunement les références de White à des hadith. Ce type de sources est particulièrement sujet à caution. Il participe souvent d'une reconstructionlégitimation de l'histoire islamique ; le fait que la tradition chi'ite attribue des étriers à Ali (White 1969: 12) confirme notre réserve. Reprenons la question des sources. Jâhiz est une source très sûre (Pellat 1953 : XI et 121). De plus, cet auteur, qui fut le disciple d'Abu'Ubayda - philologue et qui aurait été kharijite (Pellat 1953 : 143; Gibbs 1991)-, avait une certaine connaissance du persan de l'époque et a eu entre les mains des ouvrages dans cette langue (Pellat 1953:68). Enfin, Jâhiz est une source antérieure à celles d'Ibn Qutayba et de Mubarrad; ce dernier connaissait fort bien son œuvre et il est très probable que, sur la question des étriers, Mubarrad se soit directement inspiré de Jâhiz. C'est pourquoi, contre la thèse de Mubarrad attribuant au général Muhallab leur introduction dans les armées arabes, il nous semble préférable de suivre Jâhiz quand il affirme que l'adoption des étriers en métal par les Arabes date de l'époque azraqite, disons entre 670-694, donc également bien avant les dates proposées par White. Il faut ajouter que, pour Jâhiz « ni les Kharijites, ni les bédouins ne sont réputés pour leur adresse à tirer à l'arc étant à cheval » (Douillet 1977), sa référence en matière de stabilité et d'adresse dans le combat à cheval étant le cavalier turc (Marmouset 1990).

17 Récemment, Shahîd (1995), reprenant la thèse de Bivar de la diffusion des étriers des Byzantins chez les Arabes puis de ces derniers chez les Perses, ajoute que ce sont probablement les Arabes ghassanides qui furent les intermédiaires entre Byzantins et Arabes. Il s'agit pour Shahîd de conforter la thèse de Bivar. Examinons quelques 
arguments de cet auteur. Shahîd affirme que, comme chez les Byzantins, la fonction première des étriers chez les Arabes consiste, non pas à donner une bonne stabilité au cavalier, mais en une aide pour monter sur le cheval, et il précise que cela se voit dans le choix du mot arabe rikâb, dérivé du verbe rakiba qui irait dans le même sens (Shahîd 1995 : 574 et 611). Cette affirmation est pour le moins contestable car rakiba est une racine arabe qui renvoie plutôt à l'idée générale de monter, notamment sur un animal (cheval, mulet, dromadaire), et de voyager sur une monture ou un navire (Zamakhsharî 1985, I : 365). Le mot rikâb (étrier) donné par Ibn Manzur (Lisân al'-arab, racine : r. k. b.), comme par son prédécesseur Zamakhsharî, nous semble évoqué dans le sens de ce qui permet de bien voyager, c'est-à-dire d'une manière stable. Et comme nous l'avons déjà noté, Jâhiz insiste sur le fait que les étriers sont essentiels pour porter des coups efficaces (c'est-à-dire plus efficaces que sans étriers en métal) de lance, d'épée ou de sabre. Les anciens Arabes valorisaient la monte sans étriers, et le fait de bondit sur la monture était un signe de virilité. Ce qui se trouve sans aucun doute en question, c'est non pas l'aide que fournissent les étriers pour monter à cheval, mais clairement la stabilité du cavalier en vue de renforcer son efficacité dans le maniement des armes lors d'un combat à cheval. Il s'agit, chez Shahîd comme chez White ou chez Cartier, d'une même confusion de lignées technologiques différentes. Les étriers comme aideappui s'insèrent dans un certain agencement cérémoniel et esthétique lié à un haut statut : comme dans le cas des premiers « étriers » chinois dits de monte, présents mais d'un seul côté (Des chevaux et des hommes 1995 : 15), ou de la sangle d'orteil du prince indien (White 1969). Les étriers comme élément de stabilité dans le combat à cheval renvoient à un tout autre agencement, celui d'une machine de guerre propre aux nomades ou aux sédentaires. Ce sont deux lignées techno-politiques différentes, qui actualisent deux tendances, deux virtualités, elles aussi distinctes. Enfin, Shahîd (1995 : 578) ne voit pas la contradiction entre ses conclusions $n^{\circ} 1$ et $n^{\circ} 5$. À propos de la victoire des Arabes sur les Byzantins à la bataille de Yarmûk, il laisse entendre que la présence, dans les troupes arabes, de chevaux avec étriers a pu contribuer à cette victoire. Or, selon Shahîd lui-même, les Arabes, par le biais des Ghassanides, avaient emprunté les étriers peu de temps avant aux Byzantins, ces derniers les ayant euxmêmes empruntés auparavant aux Avars autour de 600: les deux cavaleries se trouvaient alors sur un pied d'égalité de ce point de vue.

Quant au fait que Mubarrad en attribue l'invention à Muhallab, cela n'a rien d'étonnant, car Muhallab est le héros de la ville de Basra. Il est celui qui a sauvé cette ville du kharijisme, et au héros, on prête souvent de nombreuses qualités (Pellat 1953 : 211), donc pourquoi pas une invention technique... Quant à la thèse de Bivar (1972) affirmant que les Perses ont connu les étriers grâce aux Arabes qui les ont empruntés aux Byzantins d'Héraclius, lesquels les avaient eux-mêmes empruntés aux Avars, elle ne tient pas assez compte des textes arabes, des données sur les nomades, ni surtout de la découverte de deux paires d'étriers sassanides -que ni lui, ni White ne pouvait, il est vrai, connaître. Le fait de n'avoir découvert que peu d'étriers d'époque sassanide s'explique soit parce que seuls certains corps de cavalerie d'élite en faisait usage ${ }^{8}$, soit parce que ces étriers ont été réutilisés par les cavaliers arabes (il n'est pas très difficile d'emprunter une selle et sa paire d'étriers et de la réinstaller sur une monture ${ }^{9}$ ), soit enfin, parce qu'ils ont été fondus et employés à d'autres fins, ou encore pour toutes ces raisons. connaissaient les étriers mais ne les employaient que d'une manière limitée pour des 
raisons culturelles et symboliques (Tanavoli 1998: 44), d'autre part que c'est bien en Perse que les Arabes, y compris bien sûr les kharijites, empruntèrent les étriers de métal, et probablement dès le début de la conquête islamique et des combats avec la cavalerie sassanide dans le Bas-Iraq. À cet égard, il est intéressant de rappeler que Baladhuri signale que lors de la bataille de Qadisiyya (635-637), 4000 soldats sassanides passèrent du côté des musulmans et qu'un important groupe de cavaliers sassanides installés à Basra rallièrent également les musulmans (Frye 1975:61). Les Arabomusulmans ayant commencé la conquête militaire de la Perse en 635-636, on a du mal à croire qu'ils auraient mis 50 ans pour trouver une paire d'étriers et en apprécier l'intérêt, alors que dès 653, il y a un gouverneur arabe au Khorassan et que commencent les accrochages avec les Turcs (Lambton 1978). Pour en revenir à Muhallab, il doit être celui qui a généralisé, systématisé la fabrication et la diffusion des étriers en métal dans les armées islamiques et/ou a fait développer un nouveau procédé de fabrication. Quant à la cavalerie sassanide, c'est aux nomades turcs d'Asie centrale présents sur les frontières de l'empire qu'elle doit l'adoption des étriers. En effet, le problème des Perses sur leur frontière, ce sont les Turcs et depuis longtemps (Chtistensen 1944: 313; Frye 1975 : 39, 49). De leur côté, les armées byzantines ont plutôt emprunté les étriers aux nomades avars, comme elles leur ont emprunté un type de mors et de flèches dites à trois ailerons (Haldon $1975: 22-23$; Bavant $1990: 229,241$ ).

\section{Conclusion}

20 Il serait présomptueux de vouloir conclure sur ce sujet difficile. Certains éléments sont établis, mais de nombreuses questions demeurent. Il faut, nous semble-t-il, abandonner l'essentiel des thèses de White, notamment l'idée que les Perses n'eurent pas connaissance des étriers et que les Arabes les introduisirent en Iran; ou encore qu'ils sont une invention chinoise, et que leur adoption par les cavaleries européennes médiévales fut l'élément décisif qui bouleversa l'ordre social en Europe -si l'on en croit Bachrach $^{10}$.

21 Une vraie question demeure, que ne posait pas White : pourquoi les cavaleries perses et byzantines, souvent en guette et en contact avec les nomades depuis des siècles, se sont-elles privées si longtemps des étriers, simples à fabriquer et aux conséquences militaires et politiques si importantes? Comment expliquer qu'elles aient mis si longtemps à les emprunter? Les combats s'engageaient-t-ils une fois les belligérants descendus de leur monture? D'autre part, s'il y a peu de doutes sur le fait que les cavaleries byzantines et perses n'ont adopté les étriers que tardivement (610-20 pour Byzance ?, 622-630 pour les armées sassanides ?), disons au cours de la première moitié du VII ${ }^{\mathrm{e}}$ siècle, les dates exactes de leur apparition au Moyen-Orient restent à préciser. De nouvelles recherches, notamment archéologiques, apporteront certainement des éléments inédits et de nouvelles réponses.

22 Anthropologues et historiens ont toujours été confrontés à des diffusions notamment technologiques, en particulier dans l'espace eurasiatique, le grand axe cher à LeroiGourhan ; c'est pourquoi une approche diffusionniste tempérée nous semble tout à fait d'actualité, quelle que soit la période historique. Claude Lévi-Strauss, pourtant si critique et méfiant à l'égard du diffusionnisme, n'écrivait-il pas à juste titre :

«Les anthropologues de l'école diffusionniste n'ont pas hésité, pour leur part, à

forcer la main à la critique historique. Je ne songe pas à défendre leurs hypothèses 
aventureuses ; mais il faut bien dire que l'attitude de leurs prudents adversaires est, dans l'ordre négatif, aussi peu satisfaisante que les prétentions fabuleuses dont ils prennent le contre-pied. [...]. Nier des faits, parce qu'on les croit incompréhensibles, est certainement plus stérile, du point de vue du progrès de la connaissance, que d'élaborer des hypothèses ; même si celles-ci sont irrecevables, elles suscitent, précisément par leur insuffisance, la critique et la recherche qui sauront un jour les dépasser. » (Lévi-Strauss 1958 : 272-273). diffusion des étriers -objets importants pour la technique équestre, la technologie militaire et la guerre. De notre point de vue, le cheminement le plus probable de cette diffusion à travers l'Eurasie fut le suivant: inventés par les nomades turcs et/ou mongols de Haute Asie et de Sibérie quelques siècles avant notre ère, les étriers se diffusèrent, et furent empruntés par la cavalerie chinoise plusieurs siècles après, ce que confirment les iconographies chinoises du IV ${ }^{e}$ siècle a p. J.-C. Puis, par diffusion vers l'ouest, suivant les routes terrestres de la soie, les étriers atteignirent, par le biais des Turcs, le monde iranien sassanide où les Arabes les adoptèrent au VII siècle ap. J.-C. Parallèlement, les Avars les diffusèrent vers le monde byzantin et l'Europe orientale.

Renoncer aux thèses de L. White Jr. sur l'origine et la diffusion des étriers ne doit pas nous interdire de rappeler l'aspect pionnier et novateur du travail de cet auteur, si rare en histoire et en anthropologie des techniques.

\section{BIBLIOGRAPHIE}

Ambroz, A.K

1973, «Les étriers et les selles du Haut Moyen-âge comme indice chronologique, IV ${ }^{\mathrm{e}}$-VIII ${ }^{\mathrm{e}}$ siècle », Sovietskaya Archeologija 4 : 81-98 (en russe, trad. privée de Mme B. Mauriac).

Bachrach, Bernard

1970, "Charles Martel, mounted shock combat, the stirrup and feudalism », Studies in Medieval and Renaissance History $7: 49-75$.

Bavant, Bernard

1990, « Harnachement et attelage », pp. 241-295, in B. Bavant, V. Kondié et J-M. Spieser (eds), Caracin Grad II. Institut Archéologique de Belgrade, Éditeur : École Française de Rome (75).

Bivar, A. D. H.

1955, « The stirrup and its origins ", Oriental Art 1, I : 61-65.

1972, "Cavalry equipment and tactics on the Euphtates frontiers », Dumbarton Oaks Papers 26 :

273-291.

Cartier, Michel

1993, "Considérations sur l'histoire du harnachement et de l'équitation en Chine », Anthropozoologica $18: 29-43$ ("Les animaux dans la culture chinoise »).

Des chevaux et des hommes

1995, Catalogue de l'exposition du Musée Guimet (19 oct. 1995-15 janv. 1996), Musée National des Arts Asiatiques-Guimet. Paris : Éditions de la Réunion des Musées Nationaux. 
Christensen, Arthur

1944, L'Iran sous les Sassanides. Annales du Musée Guimet, tome 48. Paris : Geuthner.

Clutton-Brock, Juliet

1992, A History of the Horse and the Donkey in Human Societies. Cambridge, MA : Harvard University

Press.

Deloche, Jean

1989, Military Technology in Hoysala Sculpture, XII ${ }^{e}$-XIII ${ }^{e}$. New Delhi.

1986, Le cheval et son harnachement. Paris : Éditions de l'École Française d'Extrême-Orient (EFEO).

Digard, Jean-Pierre

1987, Asb-savârî, article dans Encyclopaedia Iranica, E. Yarshater éd. Vol. II, fasc. 7, New York/

London : Éditions Routledge \& Kegan Paul.

1994, Le cheval, force de l'homme. Paris : Gallimard.

Douillet, Georges

1977 Article « Furûsiyya », in Encyclopédie de l'Islam. Leiden : E. J. Brill (2 ${ }^{\text {ème }}$ édition).

Frye, N. Richard

1975, The Golden Age of Persia. Arabs in the East. London : Weidenfeld \& Nicolson Ldt.

Gernet, Jacques

1972, Le Monde chinois. Paris : Éditions A. Colin (nouvelle édition 2000).

Gibbs, H. A. R.

1991, Article « Abu 'Ubayda », in Encyclopédie de l'Islam. Leyden : E. J. Brill (2 2 ème édition).

Haldon, J. F.

1975, « Some aspects of Byzantine military technology », Byzantine and Modern Greek Studies 1 :

11-47.

1980, « The avar-byzantine frontier in the $8^{\text {th }}$ and $9^{\text {th }}$ centuries $»$, Zbornik Radova 19.

Haudricourt, André-Georges

1936, « De l'origine de l'attelage moderne ", in La technologie, science humaine. Paris : Éditions de la Maison des Sciences de l'Homme (1987).

Herrmann, Georgina

1989, « Parthian and Sasanian saddlery. New light from the Roman West », pp. 757-809, in L. De Meyer et E. Haerinck (eds), Archaeologia Iranica et Orientalis. Miscellanea in honorem Louis Vanden Berghe, vol. II, Gent.

Hyland, Ann

1994, The Medieval Warhorse, from Byzantium to the Crusades. Dover (USA) : A. Sutton Publishing Ltd.

Ibn Manzûr

1955, Lisân al-'Arab. Le Caire : Dar al-ma'ârif.

Ibn Qutayba

1954, « Kitâb al-'arab », in Rasâ'il al-Bulaghâ’, Le Caire : M. Kurd Alic (éditeur).

Jâhiz

1968, al-Bayân wa'l-tabyin. Beyrouth : Dâr al-fikr li'l-jamî' (2 vol.).

Lambton, A. K. S.

1978, Article « Iran », in Encyclopédie de l'Islam. Leyden : E. J. Brill (2 $2^{\text {ème }}$ édition). 
Leroi-Gourhan, André

1971, L'homme et la matière. Paris : Éditions Albin Michel (« Sciences d'aujourd'hui »).

1973, Milieu et techniques. Paris : Éditions Albin Michel (« Sciences d'aujourd'hui »).

Levi-Strauss, Claude

1958, Anthropologie structurale I. Paris : Plon.

Littauer, Mary Aiken

1981, « Early Stirrups », Antiquity LV : 99-105.

L 'or des Sarmates, Entre Asie et Europe. Nomades des steppes dans l'Antiquité

1995, Catalogue publié par le Centre Culturel Abbaye de Doualas du Finistère.

Needham, Joseph

1969, La Science chinoise et l'Occident. Paris : Seuil (« Point »).

Matmouset, Aicha

1990 Kitâb al-turk (Jâhiz), traduction et commentaire. Mémoire de maîtrise. Bibliothèque arabe de l’Université Paris III.

Mubarrad

s. d., al-Kâmil fi 'l-lughat wa 'l-adah. Beyrouth : Éditions Maktabat al-Ma'ârif (reproduction de l'édition du Caire).

Overlaet, Bruno

1993, « Organisation militaire et armement », in Splendeurs des Sassanides, Catalogue de

l'exposition de Bruxelles (1993). Bruxelles : Éditions des Musées Royaux d'Art et d'Histoire.

Pellat, Charles

1953, Le Milieu basrien et la formation de Gahiz. Paris : Adrien-Maisonneuve.

Pelliot, Paul

1926, Compte-rendu de l'ouvrage du Cdt Lefebvre De Noëttes : La force animale à travers les âges, Journal Asiatique 24 : 256-268.

Rubinacci

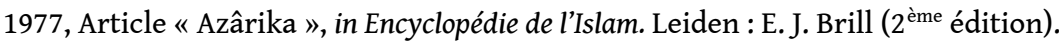

Senac, Jean

1980, Musulmans et Sarrasins dans le sud de la Gaulle du VIII au XI ${ }^{e}$ siècles. Paris : Éditions Le

Sycomore.

Shahîd, Irfan

1995, Byzantium and the Arabs in the Six Century, vol. 1, Part. 1, Political and Military History.

Washington, D.C. : Dumbarton Oaks Reseatch Library and Collection.

Schiltz, Véronique

1975, L'or des Scythes. Paris : Éditions des Musées Nationaux (Trésots des musées soviétiquesexposition du Grand Palais 8 oct-21 déc. 1975).

Splendeurs des Sassanides

1993, Catalogue de l'exposition de Bruxelles (12-02-93/25-04-93), Bruxelles.

Tanavoli, Parviz

1998, Horse and Camel Trappings from Tribal Iran. Téhéran : Éditions Farhangsara Publications.

Tolstoi, Léon

1999, Maître et serviteur. Paris : Éditions des Mille et une Nuits. 
Tseveendorj, D.

1996, « Horse and Mongols ", The Prehistory of Asia and Ocenia 16 : 89-93 (Colloquium XXXI : The evolution of nomadic herding civilisations in the northern european steppes : the tools of archaeology and history compared, A.B.A.C.O. Edizioni).

Vanden Berghe, Louis 1993, « La sculpture », pp. 71-88, in Splendeur des Sassanides. Catalogue de l'exposition de Bruxelles (12-02-93/25-04-93), Bruxelles.

Wensinck, A. J.

1993, Article « Nâfi' b. al-Azrak », in Encyclopédie de l'Islam. Leiden : E. J. Brill (2 ème édition).

White, L. Jr.

1969, «Étrier, combat à cheval et féodalité », in Technologie médiévale et transformations sociales.

Paris : Éditions de l'EHESS/Mouton (1 ère édition angl. 1962, Medieval Technology and social change,

Oxford : Oxford University Press).

Zamakhshari

1985, Asâs al-Balâgha. Le Caire : Éditions Markaz Tahqîq al-Turâth.

\section{NOTES}

2. «Le principal argument en faveur de l'origine chinoise de l'étrier est sa présence précoce dans des provinces méridionales. » (Cartier 1993 ; 33).

3. «The oldest metal stirrups, made of iron, have been found in Hiongnù burials of the third to the first centuries b.c. ».

4. Les bottes en feutre dur portées par les Russes et que mentionne Tolstoï dans sa nouvelle Maître et serviteur trouvent très certainement leur origine chez les nomades des steppes (Tolstoï 1999 : 14); en effet, la fabrication et l'utilisation du feutre sont une technique ancienne des nomades turco-mongols des steppes asiatiques (Leroi-Gourhan $1971: 240$ ). On notera également que les mots arabe et persan pour boue ; jâzma (en arabe) et cakmé (en persan) ont la même origine turque.

5. Techniques et savoirs métallurgiques sont bien maîtrisés par les nomades et vont de pair avec ceux de l'orfévrerie-bijouterie. Or, dès avant notre ère, les nomades des steppes développèrent un art fabuleux des bijoux, notamment bracelets de mains et de pieds (Schiltz 1975; L'Or des Sarmates 1995).

6. Je remercie Philippe Gignoux de ses remarques à propos du mot aspandak.

7. A. Cheikh Moussa, que je remercie, me signale que les étriers en bois chez les Arabes sont déjà mentionnés dans la poésie anté-islamique. Les étriers en bois sont également connus en Corée, en Mongolie, chez les Kirghizes et les Bashkirs (Ambroz 1973: 86-87), en Sibérie et en Russie (Littauer 1981). On les trouve aujourd'hui encore utilisés par certains gauchos argentins, comme me l'a signalé $T$. Barthélémy, que je remercie. L'existence de ce type d'étriers n'est pas mystérieuse mais correspond exactement à ce que Leroi-Gourhan appelle la réalisation de la tendance technique. Resterait à déterminer ce qui dans le milieu technique, par exemple chez les Indiens d'Amérique du Nord, n'a pas permis l'actualisation immédiate de cette tendance, aussitôt après avoir connu le cheval suite à l'arrivée des Espagnols.

8. Les armées sassanides auraient-elles réservé l'étrier aux hauts dignitaires alors que les simples cavaliers auraient continué à pratiquer la monte parthe ? Ou alors, est-ce que seul un corps de cavalerie légère aurait utilisé les étriers, comme pourrait le laisser penser le fameux bas relief de Taq-i Bustan («Scène de la chasse aux cerfs »), où les cavaliers dont le cheval est au galop ont des 
étriers alors que le roi qui assiste à la chasse (en haut du bas relief) a les jambes tombantes et une selle sans étriers?

9. Je remercie G. Douillet de m'avoir signalé que c'est ce que firent les Indiens d'Amérique du Nord.

10. La question des étriers en Occident a été discuté par Bachrach (1970). Cet auteur situe leur apparition en Occident autour de 700 et remet en cause la thèse de White quant au rapport entre l'adoption des étriers et la formation de la «classe » des chevaliers. Resterait à examiner la possibilité d'un emprunt par les armées franques à la cavalerie arabo-berbère, présente dans le sud de la France d'environ 719 à 759 (Sénac 1980 : 75, 79, 80, 82). En effet, du Moyen-Orient, et sous l'effet de l'expansion arabo-musulmane, les étriers se sont probablement diffusés vers le Maghreb et l'Andalousie musulmane. Quand les quelques corps d'armées arabo-berbères atteignirent la région de Poitiers en France, après une présence de quarante ans dans le Narbonnais, et qu'ils affrontèrent les troupes de Charles Martel, ces dernières ont tout à fait pu emprunter les étriers. Comme d'une façon tout aussi plausible, les peuples germaniques ont pu les adopter un peu auparavant et parallèlement aux armées de Charles Martel, les ayant connus lors des invasions hunniques des $\mathrm{IV}^{\mathrm{e}}-\mathrm{V}^{\mathrm{e}}$ siècles ap. J.-C. en Europe.

\section{RÉSUMÉS}

Depuis l'article écrit par Lynn White Jr. en 1962, l'invention et la diffusion des étriers en Eurasie sont considérées, soit comme des faits établis "à quelques nuances près", soit comme quasiment impossibles à cerner. Nous proposons dans cet article de nouvelles hypothèses, fondées sur des éléments inédits.

Since Lynn White jr.'s 1962 article, the invention and diffusion of stirrups in Eurasia is considered either as established fact, set aside a few particulars, or as a phenomenon almost impossible to grasp. In this contribution we put forward new hypotheses based on yet unpublished material.

Desde que Lynn White Jr escribió su artículo de 1962, el invento y la difusión de los estribos se consideran ya como hechos probados " poco más o menos ", ya como procesos casi imposibles de delimitar. Se propone en este texto nuevas hipótesis, basadas en datos inéditos.

\section{INDEX}

Mots-clés : Asie, cheval, diffusion technique, étriers, mondes arabe et iranien, technologie militaire

\section{AUTEUR}

\section{DIDIER GAZAGNADOU}

Maître de conférences au Département d'Anthropologie (UFR 3 - Université Paris VIII) et membre de l'UMR 7528 du CNRS, « Monde iranien» 